\title{
Flow Evaluation and Hemolysis Analysis of BVAD Centrifugal Blood Pump by Computational Fluids Dynamics
}

\author{
Jeerasit Bumrungpetch ${ }^{1}$, Andy Chit Tan ${ }^{1}$, Shu-Hong Liu ${ }^{2}$, Xian-Wu Luo ${ }^{2}$, Qing-Yu Wu ${ }^{3}$, Jian-Ping \\ Yuan $^{4}$, and Ming-Kui Zhang ${ }^{3}$ \\ ${ }^{1}$ Faculty of Science and Engineering, Queensland University of Technology, Brisbane, \\ QLD4001, Australia \\ ${ }^{2}$ State Key Laboratory of Hydroscience and Engineering, Tsinghua University, Beijing 100084, China \\ ${ }^{3}$ School of Medicine, Tsinghua University, Beijing 100084, China \\ ${ }^{4}$ Research Centre of Fluid machinery, Engineering and Technology, Jiangsu University, Zhenjiang, \\ Jiangsu 212013, China
}

\begin{abstract}
Computational fluid dynamics (CFD) and particle image velocimetry (PIV) are commonly used techniques to evaluate the flow characteristics in the development stage of blood pumps. CFD technique allows rapid change to pump parameters to optimize the pump performance without having to construct a costly prototype model. These techniques are used in the construction of a bi-ventricular assist device (BVAD) which combines the functions of LVAD and RVAD in a compact unit. The BVAD construction consists of two separate chambers with similar impellers, volutes, inlet and output sections. To achieve the required flow characteristics of an average flow rate of $5 \mathrm{1} / \mathrm{min}$ and different pressure heads (left $-100 \mathrm{mmHg}$ and right $-20 \mathrm{mmHg}$ ), the impellers were set at different rotating speeds. From the CFD results, a six-blade impeller design was adopted for the development of the BVAD. It was also observed that the fluid can flow smoothly through the pump with minimum shear stress and area of stagnation which are related to haemolysis and thrombosis. Based on the compatible Reynolds number the flow through the model was calculated for the left and the right pumps. As it was not possible to have both the left and right chambers in the experimental model, the left and right pumps were tested separately.
\end{abstract}

Keywords: Blood Pump, Ventricular Assist device, LVAD, BVAD, Computational fluid dynamics, artificial heart.

\section{Introduction}

Heart failure is a growing health concern worldwide with approximately 550,000 cases diagnosed annually and nearly 300,000 deaths each year. It is a debilitating condition in which the heart of a patient has decreased the pumping function. Its symptoms can be improved by pharmacological treatment, or eventual transplant. Although heart transplantation is clinically very successful, it can only benefit a small number of patients due to the limited number of donors which has remain fairly constant at about 2,100 each year (Slaughter [1]) (Roger, Go [2]) . As a result, mechanical assist devices have been used for support as a bridge to transplantation or even as a long-term therapy in some cases (Pagani, Miller [3]).

A ventricular assist device (VAD) is one of the support devices aimed at assisting the native heart to pump blood through the body, thereby reducing its workload while waiting for heart transplantation or cardiac recovery. VADs are designed to assist either the right or left ventricle, or both at once. While a left ventricular assist device (LVAD) is most commonly used and is effective in many instances, up to 50\% of LVAD recipients demonstrate clinically significant postoperative right ventricular failure. In these cases, a BVAD can effectively support both the left and right ventricles, pumping blood to the systemic and pulmonary systems.

Computational Fluid Dynamics (CFD) is an efficient fluid analysis tool which has been extensively used in the field of heart pump development since 1990s due to its ability to visualize pump flow patterns and analyze hydraulic efficiency (Behbahani, Behr [4]). CFD analysis has been adopted in heart pump research as a cost-effective tool for design optimization of heart pump (May-Newman [5]), Furthermore, CFD is also being used to evaluate and predict damage to blood(Song, Throckmorton [6, Song, Throckmorton [7]).

Received July 16 2012; revised January 25 2013; accepted for publication February 28 2013: Review conducted by Prof. Hyung-Hee Cho. (Paper number O13017S)

Corresponding author: Andy Chit Tan, Professor, a.tan@qut.edu.au

This paper was presented at $5^{\text {th }}$ International Symposium on Fluid Machinery and Fluids Engineering, October 24-27, 2012, Jeju, Korea. 
In this study, CFD was used in the development of a centrifugal BVAD. This new BVAD design has a distinctive structure as it comprises of LVAD and RVAD modules in a compact unit, each of which has its independent impeller, chamber, inlet, and outlet. Both sides of the VAD are connected together by a small passage behind the impellers, designed to create a flow passage to washout fluid stagnation and eliminate recurring flow under the impeller area. The LVAD and RVAD have the same hydraulic design but with impellers rotating at different speeds to cater for different pressures at each outlet of the pump. In order to obtain the most efficient BVAD, five designs of impeller have been developed and then compared among the designs for the most suitable design for the development of the BVAD.

The main objective for the simulation task of this heart pump is to investigate the performance of preliminarily designs of BVAD before proceeding to the development of prototype pump for experimental tests. This will lead to reducing manufacturing cost and at the same time speed up the design stage of the pump development. Therefore, in this stage, several analysis techniques were adopted to gather as much information as possible from the simulation results in both hydraulic and hemodynamic properties of the pump. Since the main target of the blood pump design is to supply enough flow and head pressure across the pump, the simulations of impellers at different rotation speeds were used to identify the operation range of each design to achieve the required head pressure. Due to the characteristic of rotary blood bump which rely on high rotation speed of impeller to build up the pressure, another main concern of the pump design is the shear stress induced hemolysis; the Eulerian approach was adopted to use as a tool to estimate the hemolysis index from the shear stress data, and was used to compare between the design to find the balance between hydraulic efficiency and blood damage. Beside the numerical information gathered from the simulation as stated above, the flow field visualization in this study was also used to identify the low velocity region and recirculation underneath the impeller and through the passage between the LVAD and RVAD which are critical regions in this BVAD design as it has high risk of stagnation and forming thrombosis.

\section{Materials and Methods}

\subsection{Fluid models}

In our BVAD, both the LVAD and RAVD have the same volute and impeller design. The outlet pressure of each side is controlled by the different rotation speeds of impellers. The flow between the LVAD and RVAD is connected by a small passage located under the impellers, which was designed to eliminate stagnation under the impellers in both the LVAD and RVAD. Through this hole, a small volume of high oxygenated blood from the LVAD will be driven to the RVAD due to the different pressures of each side; this constant flow will eliminate the chance of stagnation behind each impeller.
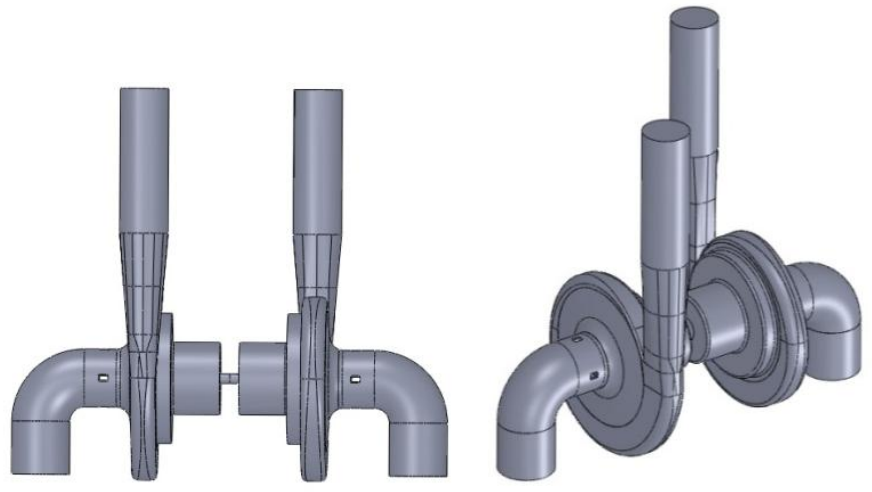

Fig. 1 Fluid model of BVAD

In this study, simulation has been made on five different designs of impellers and a fixed volute: Impeller I is a six twisted blades design, Impeller II has six cylindrical blades, Impeller III has four twisted blades, Impeller IV has four cylindrical blades, and Impeller $\mathrm{V}$ has three splitter blades (Figure 2).

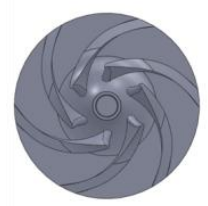

(a) Impeller I

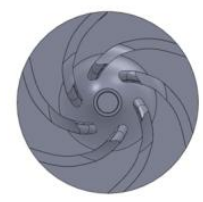

(b) Impeller II

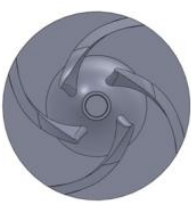

(c) Impeller III

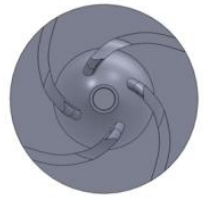

(d) Impeller IV

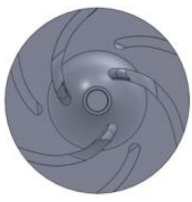

(e) Impeller V

Fig. 2 Solid models of five impeller designs 


\subsection{Simulation model and method}

The unstructured tetrahedral elements were generated on all components by means of the ANSYS 12.1 ICEM, CFX-Mesh method. An average element number for each impeller is approximately 20,000 elements and the stator is composed of 822,505 elements. The CFD simulations of these numerical models were performed in ANSYS 12.1/CFX, and the k-e model was adopted to describe the turbulence characteristic of the flow. The fluid domain was divided into three sections; composed of one stationary domain and two counter rotating domains. The interfaces between the stationary and the rotating domains were setup as frozen rotors. At the stationary domain, the inlet faces of the inlet pipes were set as the inlet boundary and the volute outlets as the outlet boundary. The non-slip condition was applied to the walls.

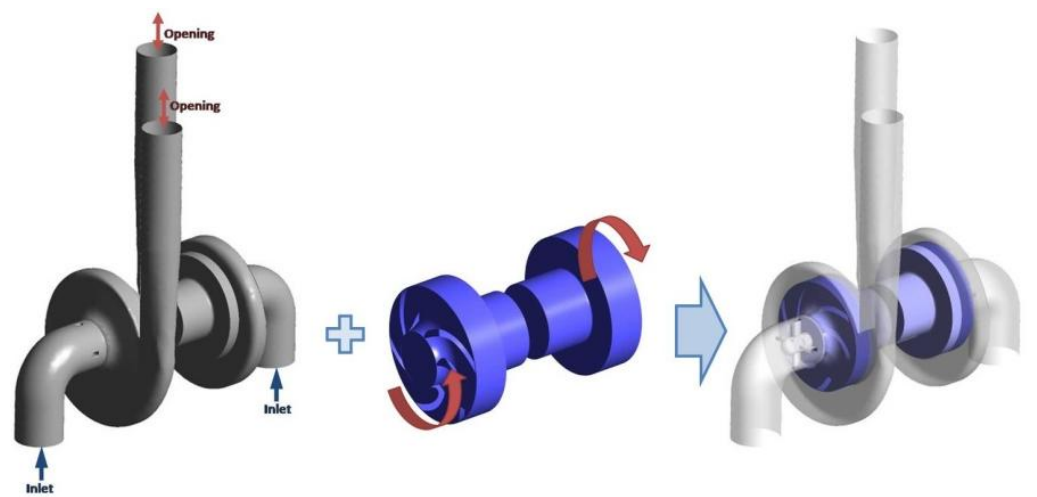

Fig. 3 Stationary and Rotating domains

Although the real blood is known to be non-Newtonian fluid, it behaves as a Newtonian fluid for shear stress greater than 100 $\mathrm{s}^{-1}$ (Day, McDaniel [8]), therefore the working fluid of rotary blood pump are normally modeled as a Newtonian fluid due to the high shear stress nature of the pumps. This assumption was experimentally validated in several literatures (Behbahani, Behr [4]) (Zhang, Gellman [9]) (Day, McDaniel [8]) and (Day, McDaniel [10]). In this study, blood was also specified to be Newtonian and have close properties to human blood with a density of $1,056 \mathrm{~kg} / \mathrm{m}^{3}$ and a constant viscosity of $0.004 \mathrm{~Pa} \cdot \mathrm{s}$. This blood-like fluid flows in each side of the BVAD with a flow rate of $0.51 / \mathrm{min}\left(0.3 \mathrm{~m}^{3} / \mathrm{h}\right)$. Due to the different working function of the VAD modules, where RVAD has to assist right ventricular system to supply venous blood to pulmonary system while LVAD supply enriched oxygen blood from left ventricle via aorta to the whole body, the required outlet pressures of the LVAD and RVAD are different. The design parameters of this pump determine the outlet pressure at $100 \mathrm{mmHg}(1.36 \mathrm{~m})$ for the LVAD and $50 \mathrm{mmHg}$ $(0.68 \mathrm{~m})$ for the RVAD. At both inlets, the pressure is set to $780 \mathrm{mmHg}$ which is the summary between the relative pressure of the blood as it enters the human heart $(20 \mathrm{mmHg})$ and the atmospheric pressure $(760 \mathrm{mmHg})$.

In order to achieve the above design parameters, simulations of the different rotation speeds have been made, then the relation of the head $(\mathrm{H})$ and the rotation speed $(\mathrm{n})$ has been plotted in the H-n graph as shown in Figure 4. By obtaining the H-n graph, the required rotation speed can be estimated as presented in Table 1.

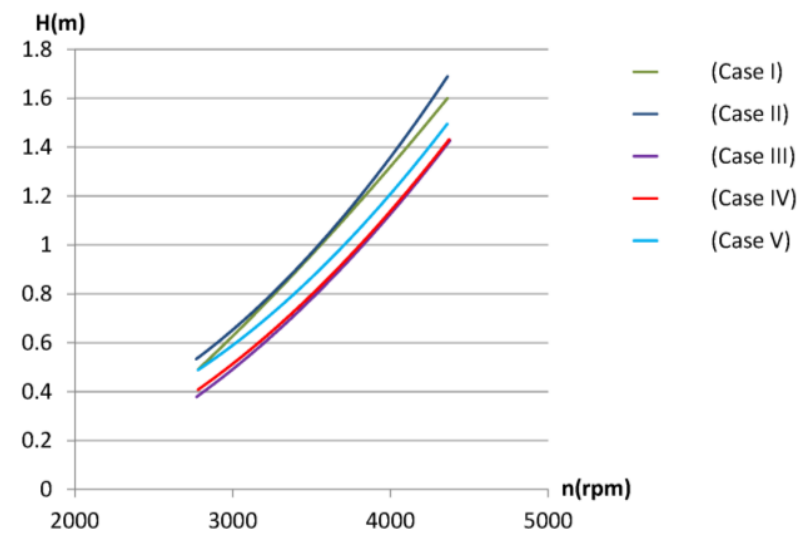

Fig. 4 H-n graph to predict the suitable rotation speed of each impeller

Table 1 Rotation speed of each case

\begin{tabular}{|l|l|l|l|l|l|}
\hline & \multicolumn{5}{|c|}{ Impeller rotation speed (rpm) } \\
\hline & Case I & Case II & Case III & Case IV & Case V \\
\hline LVAD & 4062 & 4000 & 4298 & 4283 & 4196 \\
\hline RVAD & 3111 & 3054 & 3336 & 3304 & 3181 \\
\hline
\end{tabular}




\subsection{Hemolysis Analysis}

The Eulerian approach proposed by Garon and Farinas (Garon and Farinas [11]) was adopted for the blood damage estimation in this study, and the normalized index of hemolysis (NIH) was used to compare the blood damage across the designs. According to this method, a linearized damage function is integrated over the whole domain with respect to finite volumes, yielding the average linear damage generated by the device.

$$
\overline{D_{I}}=\frac{1}{Q} \int_{V} \sigma d V
$$

where $\mathrm{Q}$ is the flow rate, $\mathrm{V}$ is the volume of the device, and $\overline{D_{I}}$ is the average linear damage.

The transformation of the linearized red blood cell damage equation from Lagrangian to Eulerian kinematics results in the following equation:

$$
\sigma=\left(3.62 \times 10^{-7}\right)^{1 / 0.785} \tau^{2.416} / 0.785
$$

The damage value $\mathrm{D}$, which is to be used in the damage index calculation is given by

$$
D=\left(\overline{D_{l}}\right)^{0.785}
$$

Finally, the NIH expressions can be evaluated by the following equations:

$$
N I H=H b \cdot D \cdot 100
$$

This blood damage model is grid independent; the accuracy of the hemolysis prediction is dependent on the accuracy of the calculation of the shear stress particularly near the wall (Medvitz, Boger [12]).

\section{Discussion and Results}

\subsection{Hydraulic Performance}

Hydraulic performance is considered to be one the most important factors in the pump design. The pump must be capable of producing enough pressure to supply blood to the pulmonary system in RVAD and the body in LVAD while maintaining high efficiency with low rotation speed to avoid excessive shear stress to the blood cell which may cause blood damage. Table 2 shows the hydraulic performance of each case under the required head and flow rate. The BVAD with impeller of Case II gives the highest hydraulic performance as it has the highest hydraulic efficiency $(55.1 \%$ for the LVAD, 53.2\% for the RVAD, and 54.5\% for the whole BVAD). Moreover this impeller requires minimum rotation speed $(4,000 \mathrm{rpm}$ for the LAVD and 3,054 rpm for the RVAD) to achieve the required specification for the pump.

Table 2 Predicted hydraulic performance from simulation

\begin{tabular}{c|cccccc}
\hline & Parameters & Case I & Case II & Case III & Case IV & Case V \\
\hline & $n_{L}(r p m)$ & 4062 & 4000 & 4298 & 4283 & 4196 \\
& $Q_{L-\text { outlet }}\left(\mathrm{m}^{3} / h\right)$ & 0.29 & 0.29 & 0.29 & 0.29 & 0.29 \\
LVAD & $H_{L}(\mathrm{~m})$ & 1.367 & 1.357 & 1.359 & 1.359 & 1.358 \\
& $P_{h}(\mathrm{~W})$ & 1.149 & 1.141 & 1.144 & 1.143 & 1.142 \\
& $\eta_{h L}$ & 0.529 & 0.551 & 0.514 & 0.522 & 0.509 \\
\hline \multirow{5}{*}{ RVAD } & $n_{R}(r p m)$ & 3090 & 3054 & 3336 & 3304 & 3181 \\
& $Q_{R-\text { outlet }}\left(\mathrm{m}^{3} / h\right)$ & 0.29 & 0.29 & 0.29 & 0.29 & 0.29 \\
& $H_{R}(\mathrm{~m})$ & 0.682 & 0.681 & 0.676 & 0.679 & 0.678 \\
& $P_{h}(\mathrm{~W})$ & 0.574 & 0.573 & 0.569 & 0.571 & 0.570 \\
& $\eta_{h R}$ & 0.511 & 0.532 & 0.487 & 0.501 & 0.496 \\
\hline \multirow{2}{*}{ BVAD } & $Q_{\text {gap }}\left(m^{3} / h\right)$ & 0.0037 & 0.0027 & 0.0025 & 0.0041 & 0.0046 \\
& $\eta_{h R}$ & 0.523 & 0.545 & 0.505 & 0.515 & 0.504 \\
\hline
\end{tabular}

\subsection{Velocity, Pressure and Shear Stress Distribution}

The flow visualization is the powerful feature of CFD simulation for flow analysis of blood pumps. The pressure distribution visualization was used in identifying the low static pressure which may lead to cavitations and resulting in corrosion of the impeller blades. In addition, the low velocity region and recurring flow which is the primary cause of thrombosis or blood clot formation can also be analyzed using the velocity and steam line distribution within the pump.

Figure 5 displays the velocity contour in the section views of the pump through three planar cuts: through inlet pipes center line of the whole pump, axial cut through center line of RVAD, and axial cut through center line of LVAD. In the coronal view of BVAD, blood flow into the inlets of both side at the same speed and with a uniform flow rate. The low velocity regions can be observed along the long radius side of the pipe elbows; it indicates that these parts should be further optimized as currently it has risk of forming thrombosis. Within the pump chamber, velocity in the volute area of LVAD is higher than RVAD due to the greater rotation speed while the flow rate under the impellers are similar, but the LVAD has slightly greater flow speed at bottom of the chamber. 

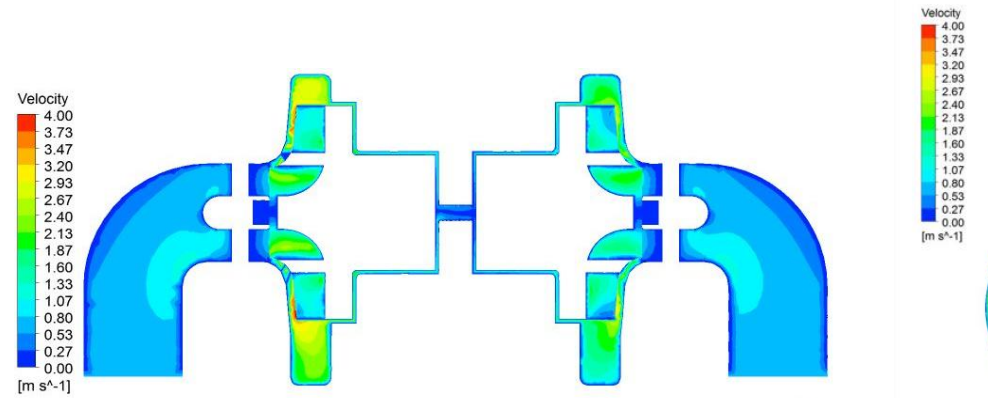


\subsection{Flow through the passage}

The small passage between the LVAD and RVAD is one of the critical points in the heart pump which is to overcome stagnation under the impellers. From the steam lines in the Figure 8, we can observe that the fluid swirls smoothly through the passage in the same rotating direction as the LVAD impeller and rotates in the reverse direction once it reaches the RVAD side (Figure 8). There is no backflow or vortex observed in the flow field. However, Figure 9 displays some small low-velocity areas under both impellers. These low velocity zones have a risk for forming thrombus and require further optimization in the future.

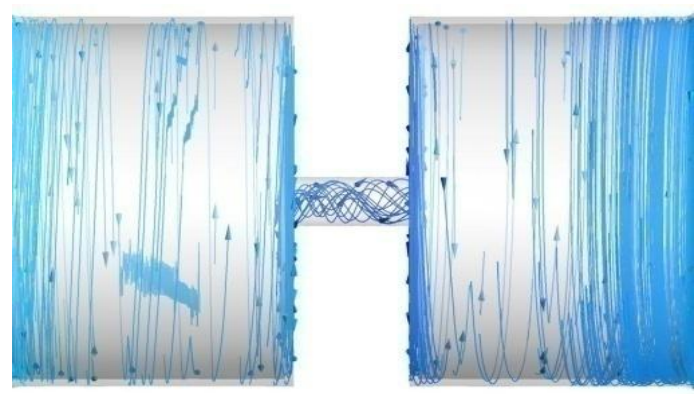

Fig. 8 Stream line through the passage

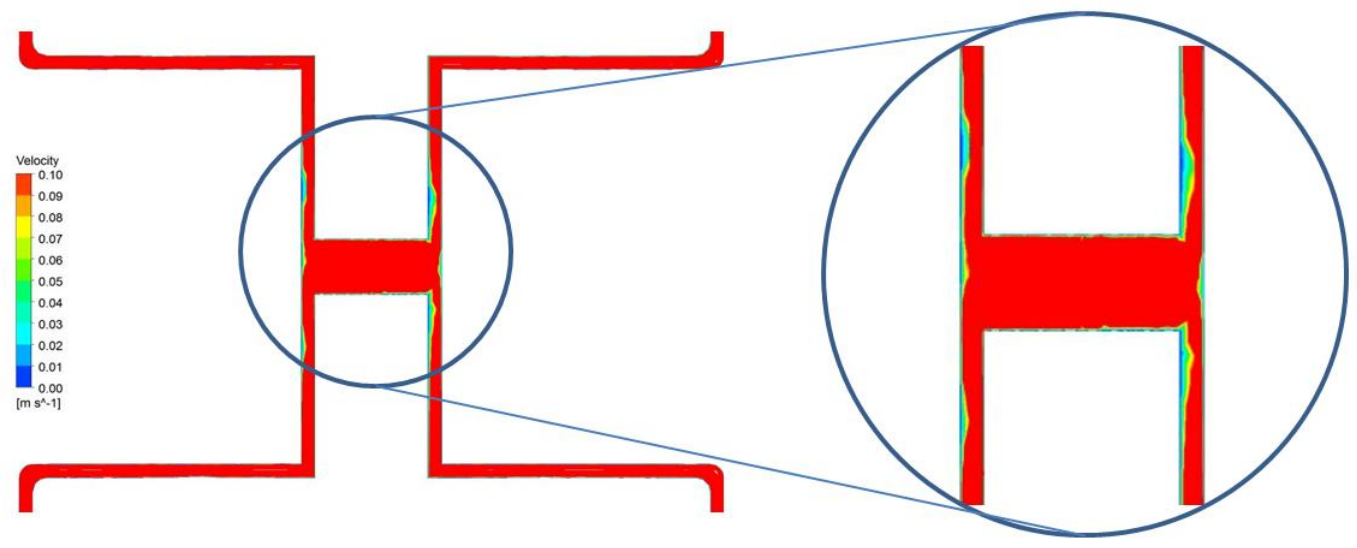

Fig. 9 Velocity contour within the passage

The blood flow rate through this passage is approximate $0.003 \mathrm{~m}^{3} / \mathrm{h}$ for all cases, and is considered to be a small amount, less than $2 \%$ of the flow rate in the device.

\subsection{Hemolysis}

Hemolysis is a destruction to red blood cell and is one of the important factors in heart pump evaluation. By obtaining the shear stress from the simulation results, both the MIH and NIH can be calculated. Table 3 displays the NIH values of each impeller compared to the limit index suggested for antitraumatic blood pump (Nosé [13]).

Table 3 NIH comparison of five cases with the clinical limit $\mathrm{NIH}(\mathrm{g} / 100 \mathrm{~L})$

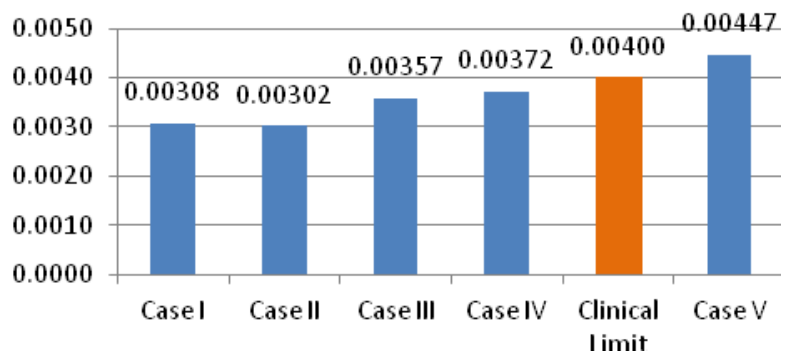


According to the graph, Cases I-IV generate a lower NIH value than the clinical limit, while Case IV causes more blood damage than the clinical acceptance. The design that generates the lowest blood damage is Case II which has an NIH value of $0.00302 \mathrm{~g} / 1001$, while Case I has slightly higher blood damage $(0.00308 \mathrm{~g} / 1001)$ than Case II, but lower than the remaining three cases.

\section{Conclusion}

The comparison of the CFD analysis results among the five hydraulic designs has exhibited that all cases have similar flow profiles. Blood can flow smoothly through the passage between the LVAD and RVAD without any vortex or stagnation in the hole. However, there are regions which have low flow rate in the bending areas of the inlet parts and under the impellers. These areas require further refinement to avoid stagnation.

According to the hydraulic performance predictions, Case II is clearly the one that provides the highest hydraulic efficiency. It also requires the lowest rotation speed to achieve the head and flow rate requirements of both the LVAD and RVAD. Case I has the second best hydraulic performance but with a lower efficiency compared with Case I, however it significantly outperformed the other three cases.

The NIH index shows that Case II is the design that generates the minimum hemolysis of $0.00302 \mathrm{~g} / 1001$, while Case I has a slightly higher NIH value of $0.00308 \mathrm{~g} / 1001$. Case V not only causes the highest blood damage but the index also exceeds the clinical limit.

Considering both the hydrodynamic and hemodynamic efficiency from the simulation results, Case II is clearly the best design in almost all aspects. It has the highest hydraulic efficiency while requiring the lowest rotation speed to reach the required flow rate and head; moreover it generates the minimum blood damage among the cases. Case I can be considered as a backup design that is also suitable for the BVAD, with its slightly lower hydraulic performance and small higher value of the hemolysis index.

The optimized design of the BVAD expressed an improvement of flow velocity under the impellers' areas; the low velocity regions under the impellers are smaller which means the risk of stagnation is also lower. As for hemolysis, with the NIH index at $0.00283 \mathrm{~g} / 1001$ or an approximate reduction of $6.3 \%$, it can be proved that the new design can reduce overall shear-stress-induced hemolysis across the pump.

On the other hand, the optimized design has some drawbacks in the hydrodynamic efficiency of the pump. As the new design allows the leakage of fluid to flow through the passage between the LVAD and the RVAD at a higher flow rate $\left(0.0041 \mathrm{~m}^{3} / \mathrm{h}\right)$, it causes more volumetric loss on the LVAD and lower overall efficiency of the whole BVAD. However, with the very small reduction of efficiency $(0.2 \%)$, this loss is negligible. In addition, there is no significant difference between the forces and torques compared with the original design.

\section{Acknowledgments}

The authors acknowledge the financial support from the Australian and the Chinese Governments through The International Science Linkage (ISL) program, DIISR No. CH090309

\section{References}

[1] Slaughter, M.S., 2010, "Introduction to LVAD Papers," Journal of Cardiac Surgery, Vol. 25, No. 4, pp. 419-420.

[2] Roger, V.L., et al., 2011, "Heart disease and stroke statistics--2011 update: a report from the American Heart Association,"

Circulation, Vol. 123, No. 4, pp. e18-e209.

[3] Pagani, F.D., et al., 2009, "Extended Mechanical Circulatory Support With a Continuous-Flow Rotary Left Ventricular Assist

Device," Journal of the American College of Cardiology, Vol. 54, No. 4, pp. 312-321.

[4] Behbahani, M., et al., 2009, "A review of computational fluid dynamics analysis of blood pumps," European Journal of Applied Mathematics, Vol. 20, No. 04, pp. 363-397.

[5] May-Newman, K., 2010, "Computational Fluid Dynamics Models of Ventricular Assist Devices", in Computational Cardiovascular Mechanics, J.M. Guccione, G.S. Kassab, and M.B. Ratcliffe, Editors. Springer US. pp. 297-316.

[6] Song, X., et al., 2003, "Computational fluid dynamics prediction of blood damage in a centrifugal pump," Artificial organs, Vol. 27, No. 10, pp. 938-941.

[7] Song, X., et al., 2004, "Quantitative Evaluation of Blood Damage in a Centrifugal VAD by Computational Fluid Dynamics," Journal of Fluids Engineering, Vol. 126, No. 3, pp. 410-418.

[8] Day, S.W., et al., 2001, "Particle Image Velocimetry Measurements of Blood Velocity in a Continuous Flow Ventricular Assist

Device," ASAIO Journal, Vol. 47, No. 4, pp. 406-411.

[9] Zhang, J., et al., 2006, "Computational and Experimental Evaluation of the Fluid Dynamics and Hemocompatibility of the CentriMag Blood Pump," Artificial Organs, Vol. 30, No. 3, pp. 168-177.

[10] Day, S.W., et al., 2002, "A Prototype HeartQuest Ventricular Assist Device for Particle Image Velocimetry Measurements," Artificial Organs, Vol. 26, No. 11, pp. 1002-1005. 
[11] Garon, A. and M.-I. Farinas, 2004, "Fast Three-dimensional Numerical Hemolysis Approximation," Artificial Organs, Vol. 28, No. 11, pp. 1016-1025.

[12] Medvitz, R.B., et al., 2011, "Computational Fluid Dynamics Design and Analysis of a Passively Suspended Tesla Pump Left Ventricular Assist Device," Artificial Organs, Vol. 35, No. 5, pp. 522-533.

[13] Nosé, Y., 1998, "Design and Development Strategy for the Rotary Blood Pump," Artificial Organs, Vol. 22, No. 6, pp. 438446. 